\title{
Transmission Electron Microscopy As A Relevant Tool In The Characterization Of Hybrid Nanostructures Of Au Bio-Mineralization By Electroactive Bacteria.
}

\author{
Muñoz, Vanesa ${ }^{1 *} ;$ Inchaurrondo, Joaquín ${ }^{1}$, Busalmen, Juan Pablo ${ }^{1}$; Ordoñez, María Victoria ${ }^{1}$ \\ 1. Institute of Materials Science and Technology (INTEMA), University of Mar del Plata and National \\ Research Council (CONICET), Colón 10890, 7600 Mar del Plata, Argentine. \\ Muñoz, Vanesa.: vanem1973@gmail.com
}

Geobacter sulfurreducens is an anaerobic gram(-) bacteria capable of using a wide range of electron acceptor, including insoluble Fe(III) (hydr)oxides, and anode electrodes, thus expelling electrons beyond cells limits. Outer membrane cytochromes (C-type) are the main redox proteins involved in external electron transfer.

We have previously observed the capacity of Geobacter to use Au(III) ions as electron acceptor, thus forming AuNPs that help improve current production in electro active biofilms. In this work we go forward on the isolation and characterization of nanostructures synthetized by Geobacter cultures grown in batch. Au respiration was performed at standard growing conditions, with 20, 100 or $200 \mathrm{mM}$ $\mathrm{AuHCl}_{4}$ for 1 week period. Soluble fraction containing gold NPs as well as biological material was then obtained and applied to a sucrose discontinuous gradient to achieve nanoparticle size separation. Both Au-containing cultures (previously fixed) and sucrose gradient fractions were placed in 200-mesh Formvar film copper grids and were studied by transmission electron microscopy in a JEOL JEM 2100 at 80 and $120 \mathrm{kV}$.

Micrographs corresponding to Geobacter cells producing AuNPs revealed the localization of such structures mostly in contact with external membrane, forming cumulus at several spots (Figure 1). Cumulus formation correlate to initial $\mathrm{Au}(\mathrm{III})$ concentration added to the stationary grown cultures. Results obtained from fractioning showed that NPs separation from cellular debris was achieved finding smaller NPs $(2-10 \mathrm{~nm})$ in $30 \%$ sucrose fraction and larger NPs in 40 to $60 \%$ sucrose fraction, most of which have an spherical shape (Figure 2). Results indicate conjugates of cytochromes-AuNPs would form, thus obtaining hybrid nanostructures. 


\section{References}

[1] Ordoñez M.V, Robuschi L, Hoppe C y Busalmen J.P Respiratory Au nucleation and microelectrode techniques reveal key features of bacterial conductive matrix. . Send to Nano Letters.Manuscript ID $\mathrm{N}^{\circ}$ nl-2019-016795. ISSN 1530-6984. (2019)
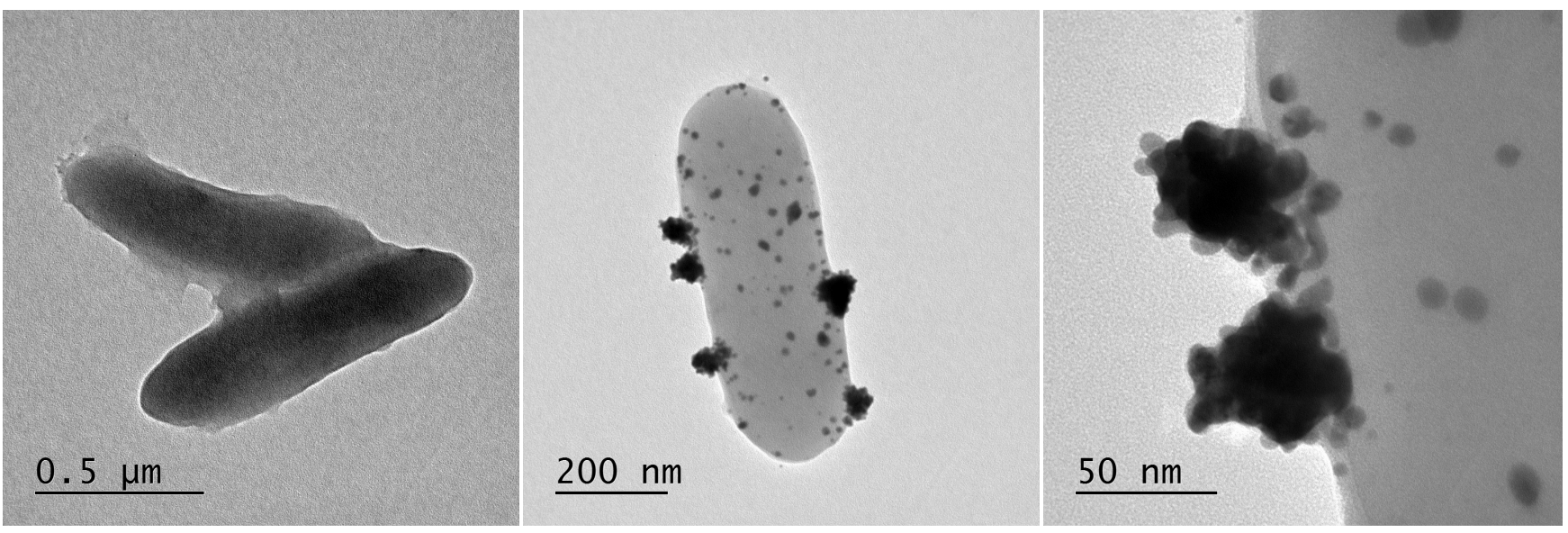

Figure 1. TEM micrographs of G. sulfurreducens cells grown in absence (A) or presence (B-C) of $\mathrm{AuHCl}_{4} 100 \mu \mathrm{M}$.
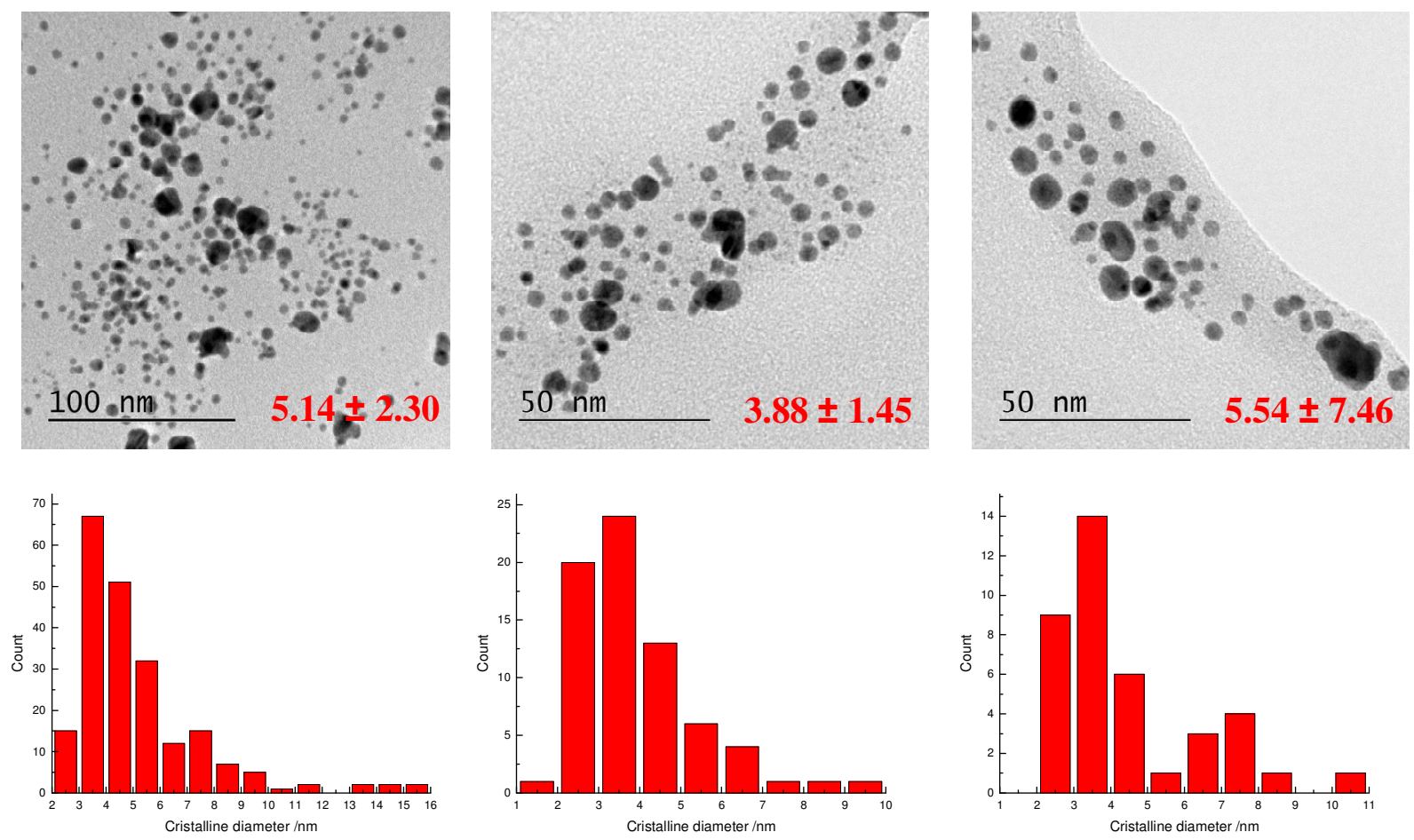

Figure 2. TEM micrographs from fractions obtained after separation in sucrose gradient. A: initial sample (total extract). B: Fraction 1 (F1) and C: Fraction 2 (F2). Diameter distribution histograms for each micrograph are shown below. 